\title{
A geometric method of sector decomposition
}

\author{
Toshiaki KANEKO \\ High Energy Accelerator Research Organization (KEK), \\ Ohe 1-1, Tsukuba, Ibaraki 305-0801, Japan \\ Takahiro UEDA \\ Graduate School of Pure and Applied Sciences, \\ University of Tsukuba, \\ Tsukuba, Ibaraki 305-8571, Japan
}

\begin{abstract}
We propose a new geometric method of IR factorization in sector decomposition. The problem is converted into a set of problems in convex geometry. The latter problems are solved using algorithms in combinatorial geometry. This method provides a deterministic algorithm and never falls into an infinite loop. The number of resulting sectors depends on the algorithm of triangulation. Our test implementation shows smaller number of sectors comparing with other existing methods with iterations.
\end{abstract}

\section{Introduction}

Analysis of recent high statistical experiments in high energy physics requires accurate theoretical predictions. Higher order corrections in perturbation theory become indispensable for the calculation of many physical processes. However, calculation of higher loop integration is not an easy problem because of its high dimensionality and complicated dependence on many kinematic and mass parameters. As the required final results of calculations are sets of numerical values, various numerical methods of loop integrations have been proposed. Since numerical method can only be applied to the quantities without divergences, one has to avoid singularities in the integrand by regularization or subtraction of divergent part before applying numerical method.

In the analysis of loop corrections in perturbative $\mathrm{QCD}, D$-dimensional regularization of infrared (IR) divergences is commonly used, where these divergences are separated in the form of poles in terms of $\epsilon=(4-D) / 2$. The method of sector decomposition [1, 2, 3, 4, 5, 6, provides a systematic procedure to extract these poles, and their coefficients are free from IR divergences. This method consists of several steps, whose details will be discussed in the next section. Here we mention each of these steps briefly:

1. Start from Feynman parameter representation of a loop integration.

2. Primary sector decomposition: 
Divide the domain of integration into sectors, eliminating the $\delta$-distribution, such that each sector includes one corner of the original integration domain.

\section{Factorization step:}

Decompose sectors repeatedly such that IR diverging part of the integrand is factored out as a product of powers of the integration variables in each sector.

\section{Divergence separation step:}

When the integrand in each sector has a divergent factor of an integration variable, one can extract the singular part expanding into Laurent series. Poles of $\epsilon$ are separated with the analytic integration of the singular part. After all poles are extracted by repeating this procedure for all divergent factors, the finite part is expanded into Taylor series in terms of $\epsilon$.

5. Integration step:

By the previous step, the coefficients of poles and the finite part are expressed by integrals of functions without IR divergences. If these functions do not suffer from other singularities, these integrations are calculated numerically. Otherwise further regulations of other singularities are necessary [7, 8, 9, 10]

The factorization step of the usual sector decomposition approach decomposes the integration domain into sectors according to a sector decomposition strategy. A strategy provides a rule to choose a subset of integration variables and to determine how chosen variables are transformed and how the domain is divided. After applying these decompositions iteratively, one will obtain integrands whose IR divergent part is correctly factored out in each resulting sector. However, it is found that some strategies may lead to an infinite loop of iteration. This problem was first solved by Ref. [11, 12, with presenting three strategies guaranteed to terminate. Their strategies are based on the analysis of the polynomials appearing in the integrand. The strategy given in Ref. [13, 14]. also solve this problem, which is based on the analysis of polytopes corresponding to the polynomials.

The number of generated sectors depends heavily on the strategies. The efficiency of sector decomposition method depends on the resulting number of decomposed sectors, since the numerical integration is the most time consuming part and will be repeated for different values of kinematic or mass parameters. Therefore such a method of the factorization step is favored that gives as small a number of generated sectors as possible.

In this paper, we propose a new method of the factorization step employing geometric interpretation of the problem. This is a deterministic method without iterated decomposition and hence never falls into an infinite loop. In section 2, the problem is rephrased in clarifying the condition of the expected output of the factorization step. The problem is converted to a set of problems in convex geometry in section 3. Latter problems are solved in section 4 with algorithms developed in combinatorial geometry. Then it is shown that the number of the decomposed sectors depends on the triangulation algorithm of $n$-dimensional 
polytopes. In section 5, we show the results of the test implementation of our method. The last section is devoted to the conclusion.

Throughout this article, we use the following 'multi-index notation'. For $m$ tuple of variables $x=\left(x_{1}, \cdots, x_{m}\right)$ and numbers $a=\left(a_{1}, \cdots, a_{m}\right)$, the product of $x_{j}^{a_{j}}$ for $j=1, \cdots, m$ is expressed by

$$
x^{a}:=x_{1}^{a_{1}} \cdots x_{m}^{a_{m}},
$$

and

$$
d^{m} x \quad:=d x_{1} \cdots d x_{m} .
$$

We also use special vector $\mathbb{I}$ defined by $\mathbb{I}:=(1, \cdots, 1)$.

\section{Sector decomposition}

Let us start with Eq. (11) of Ref. [6] for the loop integration expressed with Feynman parameters $x=\left(x_{1}, \cdots, x_{N}\right)$ :

$$
G=(-1)^{N_{\nu}} \frac{\Gamma\left(N_{\nu}-L D / 2\right)}{\prod_{j=1}^{N} \Gamma\left(\nu_{j}\right)} \int_{0}^{\infty} d^{N} x x^{\nu-\mathbb{I}} \delta\left(1-\sum_{l=1}^{N} x_{l}\right) \frac{\mathcal{U}^{N_{\nu}-(L+1) D / 2}}{\mathcal{F}^{N_{\nu}-L D / 2}},
$$

where

- $L$ is the number of loops,

- $D=4-2 \epsilon$ is the dimension of the space-time,

- $N$ is the dimension of the integration,

- $\nu_{j}(j=1, \cdots, N)$ is the power of the propagator corresponding to the Feynman parameter $x_{j}$

- $N_{\nu}:=\sum_{j=1}^{N} \nu_{j}$,

- $\mathcal{U}$ is a homogeneous polynomial of $\left\{x_{j}\right\}$ of degree $L$, and all the coefficients of the monomials of $\mathcal{U}$ are equal to 1.

- $\mathcal{F}$ is a homogeneous polynomial of $\left\{x_{j}\right\}$ of degree $L+1$, and the coefficients of the monomials of $\mathcal{F}$ consist of kinematic and mass parameters.

Polynomial $\mathcal{F}$ may approach to 0 when the vector $x$ of Feynman parameters approaches to a corner of the integration domain constrained by the $\delta$ distribution $\left(x_{j} \rightarrow 0\right.$ except for one parameter). In such cases, the integration may diverge and cause IR singularities, which are dimensionally regulated.

The primary sector decomposition step is to separate these singularities with dividing the integration domain into $N$ sub-domains such that each sector includes only one corner. Sector $l$ is defined by:

$$
\left\{\left(x_{1}, x_{2}, \cdots, x_{N}\right) \mid x_{j} \leq x_{l}, \forall j \neq l\right\} .
$$


In this sector, the integration variables are changed from $\left\{x_{1}, \cdots, x_{N}\right\}$ to $\left\{x_{l}, t_{1}, \cdots, t_{N-1}\right\}$ in the following way:

$$
\begin{aligned}
x_{j} & =x_{l} t_{j}^{\prime}, \\
t_{j}^{\prime} & :=\left\{\begin{array}{ll}
t_{j} & \text { for } j<l \\
1 & \text { for } j=l \\
t_{j-1} & \text { for } j>l
\end{array} .\right.
\end{aligned}
$$

Integrating over $x_{l}$ in Eq. (3), one obtain Eq. (14) of Ref. []:

$$
\begin{aligned}
G & =(-1)^{N_{\nu}} \frac{\Gamma\left(N_{\nu}-L D / 2\right)}{\prod_{j=1}^{N} \Gamma\left(\nu_{j}\right)} \sum_{l=1}^{N} G_{l}, \\
G_{l} & =\int_{0}^{1} d^{N-1} t t^{\nu^{\prime}-\mathbb{I}} \mathcal{U}_{l}^{\gamma}(t) \mathcal{F}_{l}^{\beta}(t),
\end{aligned}
$$

where

$$
\begin{array}{ll}
\mathcal{F}_{l}(t)=\mathcal{F}\left(t^{\prime}\right), & \beta=-\left(N_{\nu}-L D / 2\right), \\
\mathcal{U}_{l}(t)=\mathcal{U}\left(t^{\prime}\right), & \gamma=N_{\nu}-(L+1) D / 2,
\end{array}
$$

and

$$
\nu_{j}^{\prime}:=\left\{\begin{array}{ll}
\nu_{j} & \text { for } \quad j<l \\
\nu_{j+1} & \text { for } j \geq l
\end{array} .\right.
$$

After the primary sector decomposition, the domain of integration becomes $(N-$ 1)-dimensional unit cube and the corner of the original domain of integration is mapped to the origin $t_{j}=0(\forall j)$, where $\mathcal{U}_{l}$ and $\mathcal{F}_{l}$ may vanish. This expression is the starting point of our discussion.

The purpose of the factorization step is factoring out the possible singularities at $t_{j}=0$ from $\mathcal{U}_{l}$ and $\mathcal{F}_{l}$. In the usual sector decomposition approach, this factorization is obtained by dividing the domain of integration repeatedly and applying appropriate transformation of integration variables. With an appropriate strategy, one can finally find proper sub-domains and new variables suitable for the separation of IR divergences. To be more specific:

1. Integration domain is divided into $m$ sectors $\left\{D_{1}, D_{2}, \cdots, D_{m}\right\}$.

2. In each sector $D_{a}$, new variables $z=\left(z_{1}, \cdots, z_{N-1}\right)$ are introduced and $t_{j}=t_{j}(z)$ is expressed as a monomial of $z$, and Jacobian $J_{a}(z)$ is a monomial of $z$ with constant coefficient. Let monomial $z^{c_{a}}$ be a product of $t^{\nu^{\prime}-\mathbb{I}}$ and Jacobian:

$$
z^{c_{a}}=\prod_{j} z_{j}^{\left(c_{a}\right)_{j}}:=t(z)^{\nu^{\prime}-\mathbb{I}} J_{a}(z)=\prod_{k} t_{k}(z)^{\nu_{k^{\prime}}-1} J_{a}(z) .
$$

3. Integration domain of $z$ is the $(N-1)$-dimensional unit cube $\left(0 \leq z_{j} \leq 1\right)$.

4. Polynomials $\mathcal{U}_{l}$ and $\mathcal{F}_{l}$ are expressed in the following form in each sector $D_{a}$ :

$$
\begin{aligned}
& \mathcal{U}_{l}=C_{a} z^{b_{a}}\left(1+H_{a}(z)\right), \\
& \mathcal{F}_{l}=C_{a}^{\prime} z^{b_{a}^{\prime}}\left(1+H_{a}^{\prime}(z)\right),
\end{aligned}
$$


where $b_{a}$ and $b_{a}^{\prime}$ are $(N-1)$-tuples of non-negative integers $\left(b_{a}, b_{a}^{\prime} \in\right.$ $\left.\mathbb{Z}_{>0}^{N-1}\right) \sqrt{1}, H_{a}(z)$ and $H_{a}^{\prime}(z)$ are polynomials of $z$ such that $H_{a}(0)=0$ and $H_{a}^{\prime}(0)=0$.

The output of the factorization step is shown by the following form of the expression:

$$
\begin{aligned}
G_{l}=\sum_{a} C_{a}^{\beta} C_{a}^{\gamma} & \int_{0}^{1} d^{N-1} z z^{c_{a}+b_{a}^{\prime} \beta+b_{a} \gamma} \\
& \times\left(1+H_{a}(z)\right)^{\gamma}\left(1+H_{a}^{\prime}(z)\right)^{\beta} .
\end{aligned}
$$

The divergence separation step proceeds in the following way. Factor $z^{c_{a}+b_{a}^{\prime} \beta+b_{a} \gamma}$ is expressed by $\prod_{j} z_{j}^{n_{j}+m_{j} \epsilon}\left(n_{j}, m_{j} \in \mathbb{Z}\right)$. If $n_{j} \leq-1$, the integration of $z_{j}$ diverges when $\epsilon \rightarrow 0$. In this case, the integrand is split into IR divergent and finite parts. Let us consider the case of $n_{j}=-1$ for simplicity:

$$
\begin{gathered}
\int_{0}^{1} d z_{j} z_{j}^{-1+m_{j} \epsilon}\left(1+H_{a}(z)\right)^{\gamma}\left(1+H_{a}^{\prime}(z)\right)^{\beta} \\
=\int_{0}^{1} d z_{j} z_{j}^{-1+m_{j} \epsilon}\left(1+\left.H_{a}(z)\right|_{z_{j}=0}\right)^{\gamma}\left(1+\left.H_{a^{\prime}}(z)\right|_{z_{j}=0}\right)^{\beta} \\
+\int_{0}^{1} d z_{j} z_{j}^{-1+m_{j} \epsilon}\left[\left(1+H_{a}(z)\right)^{\gamma}\left(1+H_{a^{\prime}}(z)\right)^{\beta}\right. \\
\left.-\left(1+\left.H_{a}(z)\right|_{z_{j}=0}\right)^{\gamma}\left(1+\left.H_{a^{\prime}}(z)\right|_{z_{j}=0}\right)^{\beta}\right] .
\end{gathered}
$$

The first term is integrated to

$$
\frac{1}{m_{j} \epsilon}\left(1+\left.H_{a}(z)\right|_{z_{j}=0}\right)^{\gamma}\left(1+\left.H_{a^{\prime}}(z)\right|_{z_{j}=0}\right)^{\beta},
$$

and the integrand of the second term is finite for $z_{j} \rightarrow 0$. When $n_{j}<-1$, it is necessary to expand $\left(1+H_{a}(z)\right)^{\gamma}\left(1+H_{a^{\prime}}(z)\right)^{\beta}$ around $z_{j}=0$ up to the necessary order for the separation of the IR divergent parts. Repeating this procedure for all divergent integrals, we can extract all IR divergent parts as poles in terms of $\epsilon$. Then we can freely expand integrands of remaining integrals with respect to $\epsilon$, and obtain a Laurent series of the whole integral in terms of $\epsilon$, whose coefficients are expressed by IR finite integrals. We can calculate these IR finite integrals numerically if there are no other singularities in the integrands.

Let us go back to the factorization step. In order to see the problem in a different way, let us apply the expression (14) to a simple polynomial case of three variables $t_{1}, t_{2}, t_{3}$ :

$$
\mathcal{F}_{l}(t)=-s_{12} t_{1}-s_{23} t_{2} t_{3}-s_{4} t_{1} t_{3} .
$$

When the integration variables are changed to $z_{1}, z_{2}, z_{3}$ by

$$
t_{1}=z_{1} z_{2} z_{3}, \quad t_{2}=z_{1}, \quad t_{3}=z_{2},
$$

\footnotetext{
${ }^{1}$ In this paper, the set of non-negative integers and the set of non-negative real numbers are denoted by $\mathbb{Z}_{>0}, \mathbb{R}_{>0}$, respectively.
} 
we obtain

$$
\mathcal{F}_{l}(t(z))=z_{1} z_{2}\left(-s_{23}-s_{4} z_{2} z_{3}-s_{12} z_{3}\right) .
$$

This expression implies that around the origin of the domain $\left\{\left(t_{1}(z), t_{2}(z), t_{3}(z)\right) \mid 0 \leq\right.$ $\left.z_{i} \leq 1\right\}$, the term $s_{23} t_{2} t_{3}=s_{23} z_{1} z_{2}$ dominates to the others and the ratios of other terms to the dominant one become monomials of the new variables $z_{i}$. Similarly, changing variables in Eq. (18) as

$$
t_{1}=z_{1}, \quad t_{2}=z_{1} z_{2}, \quad t_{3}=z_{3},
$$

gives us

$$
\mathcal{F}_{l}(t(z))=z_{1}\left(-s_{12}-s_{23} z_{2} z_{3}-s_{4} z_{3}\right)
$$

and this exhibits a case that $s_{12} t_{1}=s_{12} z_{1}$ is dominant at the origin. Note that $s_{4} t_{1} t_{3}$ cannot be a dominant term at the origin because always $\left|s_{12} t_{1}\right|>\left|s_{4} t_{1} t_{3}\right|$ when all $t_{j}$ approach to 0 .

Above example shows that the factorization step is equivalent to solve the following set of problems:

1. to determine which terms can be dominant,

2. to determine the sub-domain where each term becomes dominant, and

3. to find a new parameterization of the variables such that other terms are expressed as monomials multiplied by the dominant term.

In the following sections, we will show that these problems can be solved by a new method without iterations.

\section{Geometric method}

Let us change the integration variables from $t=\left\{t_{1}, \cdots, t_{N-1}\right\}$ to $y=\left\{y_{1}, \cdots, y_{N-1}\right\}$ in Eq. (8) as the following:

$$
t_{j}=e^{-y_{j}} \quad \text { or } \quad y_{j}=-\log t_{j} \quad(j=1, \cdots, N-1) .
$$

Jacobian is $e^{-\sum_{j} y_{j}}$ and the integration domain is changed from $0<t_{j}<1$ to $0<y_{j}<\infty$. We obtain

$$
G_{l}=\int_{0}^{\infty} d^{N-1} y e^{-\left(\nu^{\prime}, y\right)} \mathcal{U}_{l}^{\gamma}\left(e^{-y}\right) \mathcal{F}_{l}^{\beta}\left(e^{-y}\right),
$$

where $\left(\nu^{\prime}, y\right)$ is the inner product defined in $(N-1)$-dimensional Euclidean space.

A monomial $t^{b}=t_{1}^{b_{1}} t_{2}^{b_{2}} \cdots t_{N-1}^{b_{N-1}}$ is characterized by an integer vector $b=$ $\left\{b_{1}, \cdots, b_{N-1}\right\}\left(b_{j} \in \mathbb{Z}_{\geq 0}^{N-1}\right)$. Since a polynomial $P$ is a sum of monomials, the polynomial corresponds to a finite set of such vectors $Z^{P} \subset \mathbb{Z}_{\geq 0}^{N-1}$. In terms of the new variables $\left\{y_{j}\right\}, P$ is expressed by:

$$
P(t)=\sum_{b \in Z^{P}} a_{b} t^{b}=\sum_{b \in Z^{P}} a_{b} e^{-(b, y)} \quad\left(a_{b} \neq 0, \forall b \in Z^{P}\right) .
$$


Let us consider the limit $\lambda \rightarrow+\infty$ for the variables $y_{j}=\lambda u_{j} \rightarrow+\infty(\forall j)$ with a constant vector $u \in \mathbb{R}_{\geq 0}^{N-1} \backslash\{0\} 2$ The dominant term of $P(t)$ in this limit is the monomial $a_{b} t^{b}$ whose value of $(b, u)$ takes the minimum value of $(c, u)$ among all $c \in Z^{P}$ :

$$
(b, u)=\min \left\{(c, u) \mid c \in Z^{P}\right\} .
$$

In other words, a monomial for a vector $b$ is dominant in the domain $\Delta_{b}^{P}$ of $y$ space, which is defined by:

$$
\Delta_{b}^{P}:=\quad\left\{y \in \mathbb{R}_{\geq 0}^{N-1} \mid(b, y) \leq(c, y), \forall c \in Z^{P}\right\} .
$$

Evidently the union of all $\Delta_{b}^{P}$ covers whole integration domain. The polynomial $P$ of Eq. (25) is rewritten to:

$$
P(t(y))=\sum_{b \in Z^{P}} \theta\left(y \in \Delta_{b}^{P}\right) e^{-(b, y)}\left[a_{b}+\sum_{c \in Z^{P} \backslash\{b\}} a_{c} e^{-(c-b, y)}\right] .
$$

Since $(c-b, y) \geq 0$ for $y \in \Delta_{b}^{P}$, the behavior of $P$ in the limit of $\|y\| \rightarrow \infty$ is determined by the term $a_{b} e^{-(b, y)}$. If a monomial corresponding to $b$ cannot be a dominant term, $\Delta_{b}^{P}$ is the empty set. Including this case, when the dimension of $\Delta_{b}^{P}$ is less than $N-1$, the $(N-1)$-dimensional volume of $\Delta_{b}^{P}$ is zero. We can ignore such a sub-domain, since it does not contribute to the integration. Applying the above discussion to $\mathcal{U}_{l}$ and $\mathcal{F}_{l}$ for $P$, we obtain:

$$
\begin{aligned}
G_{l}= & \sum_{b \in Z^{\mathcal{U}_{l}}} \sum_{b^{\prime} \in Z^{\mathcal{F}_{l}}} \int_{0}^{\infty} d^{N-1} y \theta\left(y \in \Delta_{b b^{\prime}}\right) e^{-\left(\nu^{\prime}+\gamma b+\beta b^{\prime}, y\right)} \\
& \times\left[1+\sum_{c \in Z^{\mathcal{U}_{l} \backslash\{b\}}} e^{-(c-b, y)}\right]^{\gamma}\left[a_{b^{\prime}}+\sum_{c^{\prime} \in Z^{\mathcal{F}_{l} \backslash\left\{b^{\prime}\right\}}} a_{c^{\prime}} e^{-\left(c^{\prime}-b^{\prime}, y\right)}\right]^{\beta},
\end{aligned}
$$

where

$$
\Delta_{b b^{\prime}}:=\Delta_{b}^{\mathcal{U}_{l}} \cap \Delta_{b^{\prime}}^{\mathcal{F}_{l}}
$$

Our first and second problems are solved when we find how to construct $\Delta_{b b^{\prime}}(\forall b \in$ $\left.Z^{\mathcal{U}_{l}}, \quad \forall b^{\prime} \in Z^{\mathcal{F}_{l}}\right)$.

These problems are interpreted in the words of convex geometry. We introduce several terms and notations according to the appendix of Ref. 15. The convex polyhedral cone for a finite set $S$ in $n$-dimensional Euclidean space is defined by:

$$
C(S):=\left\{\sum_{v \in S} r_{v} v \in \mathbb{R}^{n} \mid r_{v} \in \mathbb{R}_{\geq 0}, \forall v \in S\right\} .
$$

It is noted that a line is a convex polyhedral cone for $S=\{v,-v\}$ with a fixed vector $v$. Similarly, it is easy to see that an $m$-dimensional linear subspace $\mathbb{R}^{m}(m \leq n)$ is also a convex polyhedral cone.

${ }^{2} A \backslash B=\{a \in A \mid a \notin B\}$. Do not confuse with Minkowsky sum $A-B=A+(-B)$ defined by Eq. 45 
The dual cone of a convex polyhedral cone $C$ is defined by :

$$
C^{\vee}:=\left\{y \in \mathbb{R}^{n} \mid(v, y) \geq 0, \forall v \in C\right\} .
$$

For a finite set $S, C(S)^{\vee}$ is also a convex polyhedral cone.

Let $Z_{b}^{P}$ be the set of points translated from $Z^{P}$ such that point $b$ is moved to the origin:

$$
Z_{b}^{P}:=\left\{c-b \in \mathbb{Z}^{N-1} \mid c \in Z^{P}\right\} .
$$

Comparing with Eqs. (27) and (32), we have

$$
\Delta_{b}^{P}:=C\left(Z_{b}^{P}\right)^{\vee} \cap \mathbb{R}_{\geq 0}^{N-1},
$$

and

$$
\Delta_{b b^{\prime}}=C\left(Z_{b}^{\mathcal{U}_{l}}\right)^{\vee} \cap C\left(Z_{b^{\prime}}^{\mathcal{F}_{l}}\right)^{\vee} \cap \mathbb{R}_{\geq 0}^{N-1} .
$$

Thus what we have to solve is to find how to construct cones $\left(C\left(Z_{b}^{\mathcal{U}_{l}}\right)\right.$ and $\left.C\left(Z_{b^{\prime}}^{\mathcal{F}_{l}}\right)\right)$, dual cones $\left(C\left(Z_{b}^{\mathcal{U}_{l}}\right)^{\vee}\right.$ and $\left.C\left(Z_{b^{\prime}}^{\mathcal{F}_{l}}\right)^{\vee}\right)$, the intersections of dual cones $\left(\Delta_{b b^{\prime}}\right)$ and to see whether their volume is zero or not. The algorithms to solve these problems are developed in combinatorial geometry, which will be discussed in the next section.

Fig. 11 shows a simple example for the case of two variables:

$$
P(t)=t_{1} t_{2}^{5}+t_{1}^{2} t_{2}^{3}+t_{1}^{3} t_{2}^{2}+t_{1}^{5} t_{2}
$$

Fig. 1(a) and (b) show $Z^{P}, Z_{b}^{P}$, respectively. Vectors $\alpha, \beta$ and $\gamma$ satisfies the condition $(\alpha, v-b) \geq 0,(\beta, v-b) \geq 0,(\beta, v-c) \geq 0$ and $(\gamma, v-c) \geq 0$ for all $v \in Z^{P}$. Fig. 1(c) shows the resultant sub-domains.

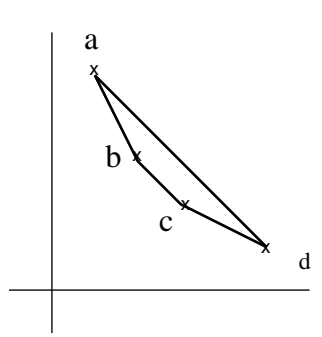

(a)

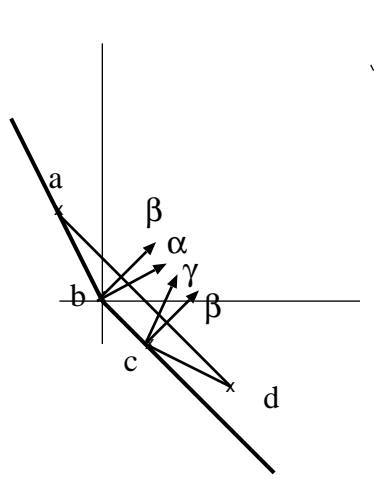

(b)

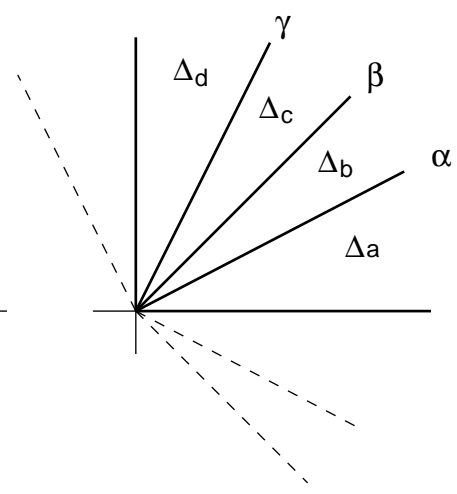

(c)

Figure 1: An example of cone and dual cone

Since these cones are in general still too complex for the integration, we divide them into a set of simpler objects. In the case of polytope (bounded 
polyhedron), it can be decomposed as a union of simplicies, which is called triangulation . The algorithms of triangulation is also studied in combinatorial geometry. Similarly, a cone can be decomposed as a union of simplicial cones as discussed in the next section.

An $(N-1)$-dimensional cone $C(V)$ is simplicial when $V=\left\{v_{1}, \cdots, v_{N-1}\right\}$ is a set of $(N-1)$ linear independent vectors. Let $S_{b b^{\prime}}$ be the set of $(N-1)$ vectors characterizing simplicial cones such that

$$
\Delta_{b b^{\prime}}=\bigcup_{V \in S_{b b^{\prime}}} C(V)
$$

represents a triangulation of $\Delta_{b b^{\prime}}$. With this triangulation, Eq. (29) is expressed by:

$$
\begin{aligned}
G_{l}= & \sum_{b \in Z^{\mathcal{U}_{l}}} \sum_{b^{\prime} \in Z^{\mathcal{F}_{l}}} \sum_{V \in S_{b b^{\prime}}} \int_{0}^{\infty} d^{N-1} y \theta(y \in C(V)) e^{-\left(\nu^{\prime}+\gamma b+\beta b^{\prime}, y\right)} \\
& \times\left[1+\sum_{c \in Z^{\mathcal{U}_{l} \backslash\{b\}}} e^{-(c-b, y)}\right]^{\gamma}\left[a_{b^{\prime}}+\sum_{c \in Z^{\mathcal{F}_{l}} \backslash\left\{b^{\prime}\right\}} a_{c} e^{-\left(c-b^{\prime}, y\right)}\right]^{\beta} .
\end{aligned}
$$

A point $y=\left(y_{i}\right) \in C(V)$ is parameterized by barycentric coordinate:

$$
y_{i}=\sum_{j=1}^{N-1}\left(v_{j}\right)_{i} u_{j}, \quad\left(u_{j} \in \mathbb{R}_{\geq 0}, \quad j=1, \cdots, N-1\right) .
$$

Since $\left\{v_{j}\right\}$ is a set of $(N-1)$ linear independent vectors, the correspondence between $\left\{y_{i}\right\}$ and $\left\{u_{j}\right\}$ is one-to-one. We regard $V=\left(v_{1}, \cdots, v_{N-1}\right)$ being a $(N-1) \times(N-1)$ matrix made arranging column vectors $\left\{v_{j}\right\}$. Point $y$ is expressed by $y=V u$ in this matrix notation. We subsequently change the variable from $y$ to $u$ then from $u$ to $z$, where $z_{j}=e^{-u_{j}}$. Jacobian is $|\operatorname{det} V| / \prod_{j} z_{j}$. We finally obtain:

$$
\begin{aligned}
& G_{l}=\sum_{b \in Z^{\mathcal{U}_{l}}} \sum_{b^{\prime} \in Z^{\mathcal{F}_{l}}} \sum_{V \in S_{b b^{\prime}}}|\operatorname{det} V| \int_{0}^{1} d^{N-1} z \prod_{j=1}^{N-1} z_{j}^{\left(\nu^{\prime}+\gamma b+\beta b^{\prime}, v_{j}\right)-1} \\
& \times\left[1+\sum_{c \in Z^{\mathcal{U}_{l} \backslash\{b\}}} \prod_{j=1}^{N-1} z_{j}^{\left(c-b, v_{j}\right)}\right]^{\gamma}\left[a_{b^{\prime}}+\sum_{c \in Z^{\mathcal{F}_{l}} \backslash\left\{b^{\prime}\right\}} a_{c} \prod_{j=1}^{N-1} z_{j}^{\left(c-b^{\prime}, v_{j}\right)}\right]^{\beta} .
\end{aligned}
$$

The original variables are expressed by:

$$
t_{j}=e^{-y_{j}}=e^{-(V u)_{j}}=\prod_{k} z_{k}^{\left(v_{k}\right)_{j}}
$$

with Jacobian $|\operatorname{det} V| \prod_{j} t_{j} / \prod_{k} z_{k}$.

As it will be shown in the next section, we can take vectors $\left\{v_{j}\right\}$ on the integer lattice $v_{j} \in \mathbb{Z}_{\geq 0}^{N-1} \backslash\{0\}$. Since $(c-b, y) \geq 0$ for all $y \in \Delta_{b}^{P}$ and $(c-b) \in \mathbb{Z}^{N-1}$, $\left(c-b, v_{j}\right)$ is non-negative integer. Thus the sub-expressions in the brackets of Eq. (40) are polynomials of $z$.

For fixed $k$, let us consider the scale transformation of vector $v_{k}$ and variable $z_{k}$ defined by $v_{k} \rightarrow \lambda_{k} v_{k}$ and $z_{k} \rightarrow z_{k}^{1 / \lambda_{k}}$ for $\lambda_{k}>0$. It is easy to see that 
integration $G_{l}$ is invariant under this transformation. We select $v_{k}$ on the integer lattice as nearest possible to the origin.

Now all the problems are converted to the following ones in convex and combinatorial geometry:

1. to construct intersections of cones $\Delta_{b b^{\prime}}=C\left(Z_{b}^{\mathcal{U}_{l}}\right)^{\vee} \cap C\left(Z_{b^{\prime}}^{\mathcal{F}_{l}}\right)^{\vee} \cap \mathbb{R}_{\geq 0}^{N-1}$ for all $b \in Z^{\mathcal{U}_{l}}$ and $b^{\prime} \in Z^{\mathcal{F}_{l}}$.

2. to triangulate $\Delta_{b b^{\prime}}$ into $\cup_{V \in S_{b b^{\prime}}} C(V)$.

\section{Algorithms}

\subsection{Construction of cones}

First we rewrite $\Delta_{b b^{\prime}}$ into a different form. The following relations hold for convex polyhedral cones $C, C_{1}$ and $C_{2}$, and for sets of finite points $S_{1}$ and $S_{2}$ (see. Ref. [15] Appendix):

$$
\begin{aligned}
C\left(S_{1} \cup S_{2}\right) & =C\left(S_{1}\right)+C\left(S_{2}\right), \\
C^{\vee \vee} & =C, \\
C_{1} \vee \cap C_{2} \vee & =\left(C_{1}+C_{2}\right)^{\vee},
\end{aligned}
$$

where addition '+' represents Minkowsky sum defined by:

$$
C_{1}+C_{2}:=\left\{a+b \mid a \in C_{1}, b \in C_{2}\right\} .
$$

Let $E^{m}$ be the standard basis of Euclidean space $\mathbb{R}^{m}$, that is

$$
\begin{aligned}
& E^{m}:=\left\{e_{1}, e_{2}, \cdots, e_{m}\right\} \\
& e_{1}=(1,0,0, \cdots, 0), e_{2}=(0,1,0, \cdots, 0), \cdots
\end{aligned}
$$

Set $\mathbb{R}_{\geq 0}^{m}$ is represented by:

$$
\mathbb{R}_{\geq 0}^{m}=\left(\mathbb{R}_{\geq 0}^{m}\right)^{\vee}=C\left(E^{m}\right)=C\left(E^{m}\right)^{\vee} .
$$

With these relations, Eq. (35) is rewritten to:

$$
\begin{aligned}
\Delta_{b b^{\prime}} & =C\left(Z_{b}^{\mathcal{U}_{l}}\right)^{\vee} \cap C\left(Z_{b^{\prime}}^{\mathcal{F}_{l}}\right)^{\vee} \cap C\left(E^{N-1}\right)^{\vee} \\
& =\left\{C\left(Z_{b}^{\mathcal{U}_{l}}\right)+C\left(Z_{b^{\prime}}^{\mathcal{F}_{l}}\right)+C\left(E^{N-1}\right)\right\}^{\vee} \\
& =C\left(Z_{b}^{\mathcal{U}_{l}} \cup Z_{b^{\prime}}^{\mathcal{F}_{l}} \cup E^{N-1}\right)^{\vee} .
\end{aligned}
$$

Thus we obtain

$$
\Delta_{b b^{\prime}}=C\left(Z_{b b^{\prime}}\right)^{\vee}
$$

where

$$
Z_{b b^{\prime}}:=Z_{b}^{\mathcal{U}_{l}} \cup Z_{b^{\prime}}^{\mathcal{F}_{l}} \cup E^{N-1} .
$$

What we should know is how to construct a convex polyhedral cone for a given finite set and how to construct its dual cone. 
Let us introduce some terms used in convex geometry. A convex hull of a subset $T$ of $\mathbb{R}^{m}$ is the smallest convex set including $T$ and is denoted by $\operatorname{conv}(T)$. When $T$ is a finite set of half-lines starting from the origin, $\operatorname{conv}(T)$ is a convex polyhedral cone. When $T$ is a finite set of points, $\operatorname{conv}(T)$ is a polytope. When $T$ is equal to the set $Z^{P}$ corresponding to a polynomial $P, \operatorname{conv}\left(Z^{P}\right)$ is called Newton polytope of $P$, which plays important role for analyzing properties of a multi-variate polynomial.

Let $K$ be a $k$-dimensional polytope or convex polyhedral cone in $m$-dimensional Euclidean space. A hyperplane $H$ is called to support a set $K$ at a point $x \in K$ if $x \in H$ and $K$ is included in one of the two closed half-spaces limited by $H$. That is, when a vector $u$ is normal to $H$, either $(u, y-x) \geq 0(\forall y \in K)$ or $(u, y-x) \leq 0(\forall y \in K)$ is true. A subset $F$ of $K$ is called a faces of $K$ if either $F=\emptyset$ or $F=K$, or if there exists a supporting hyperplane $H$ of $K$ such that $F=H \cap K$. A face of convex polyhedral cone or polytope is also a convex polyhedral cone or a polytope, respectively. A face is called a vertex, an edge or a facet when it is $0-, 1$ - or $(k-1)$-dimensional, respectively. The set of $j$-dimensional faces is denoted by $\mathcal{F}_{j}(K)$ and the set of all faces by $\mathcal{F}(K):=\{\emptyset\} \cup_{j=0}^{k} \mathcal{F}_{j}(K)$. In the case of a convex polyhedral cone, $\mathcal{F}_{0}$ is $\{0\}$ if $\mathcal{F}_{0} \neq \emptyset$. It is noted that some special convex polyhedral cones, such as lines or the whole space, have no vertices. A face of a polytope is expressed by a set of vertices included in the face. On the other hand, an edge of convex polyhedral cone is expressed by a non-zero vector laying on the edge. A higher than onedimensional face is expressed by a set of edges included in the face. Let $f$ and $g$ be two faces of $K$. When $f$ is a face of $g$, this relation is denoted by $f \prec g$. The set $\mathcal{F}(K)$ forms an abstract complex structure in terms of this binary relation $\prec$ (see. Ref. 15] Proposition A.5 and A.16).

The convex hull problem of a polytope is to find $\mathcal{F}(\operatorname{conv}(S))$ for a set of finite points $S$. This problem is popular and well studied in computational geometry (see, for example, Ref. [16]). Several software packages have also been developed to solve these problems [17, 18.

Our problem is to construct an algorithm to find $\mathcal{F}(C(S))$ for a convex polyhedral cone $C(S)$. There will be two approaches. One is to modify directly an algorithm for a polytope to one for a convex polyhedral cone. It is not so difficult and we have prepared one for our test program based on the algorithm described in Ref. [16]. The other is to use the output of algorithms for polytopes. We discuss here the latter approach.

Let us consider a correspondence between a convex polyhedral cone $C(S)$ and a polytope $\operatorname{conv}(S)$ defined for the same set of finite points $S \subset \mathbb{R}^{m}$. The polytope $\operatorname{conv}(S)$ is expressed by:

$$
\operatorname{conv}(S)=\left\{\sum_{u \in S} \lambda_{u} u \mid \lambda_{u} \geq 0, \sum_{u \in S} \lambda_{u}=1\right\}
$$

Since our set of points $Z_{b b^{\prime}}$ includes the origin 0 , we assume that $0 \in S$. Let $\mathcal{F}(\operatorname{conv}(S))$ be the set of faces obtained by a convex hull algorithm for a polytope. Comparing (31) with (52), $C(S)$ is expressed by:

$$
\begin{aligned}
C(S) & =\left\{\lambda x \mid x \in \operatorname{conv}(S), \lambda \in \mathbb{R}_{\geq 0}\right\} \\
& =\left\{\sum_{v \in \mathcal{F}_{0}(\operatorname{conv}(S))} \lambda_{v} v \mid \lambda_{v} \in \mathbb{R}_{\geq 0}, \forall v \in \mathcal{F}_{0}(\operatorname{conv}(S))\right\} .
\end{aligned}
$$


Although $S$ includes the origin, it is possible that the origin is not a vertex of $\operatorname{conv}(S)$. In this case, there exists a line segment $L$ passing through the origin and being included in both $\operatorname{conv}(S)$ and $C(S)$. Let the line segment $L$ be expressed by $L=\{\lambda v \mid a \leq \lambda \leq b\}$ with some non-zero vector $v$ and real numbers $a<0$ and $b>0$. For any $y \in C(S)^{\vee}$, condition $\lambda(v, y) \geq 0$ is satisfied for both positive and negative value of $\lambda$, and then $(v, y)=0$. So $C(S)^{\vee}$ is included in a hyperplane perpendicular to vector $v$. It means that the dimension of $C(S)^{\vee}$ is less than $m$, and this sub-domain does not contribute to the integration in our problem.

Now we assume the origin is a vertex of $\operatorname{conv}(S)$. Then we can assume the origin is also a vertex of $C(S)$. If not so, any hyperplane passing through the origin does not support $C(S) \operatorname{nor} \operatorname{conv}(S)$, which is a contradiction to the first assumption. These convex polyhedral cones are called strongly convex. Let $\mathcal{F}_{j}(C(S))$ be the set of $j$-dimensional faces of $C(S)$ and $\mathcal{F}_{j}^{\prime}(\operatorname{conv}(S))$ be the set of $j$-dimensional faces of $\operatorname{conv}(S)$ including the origin : $\mathcal{F}_{j}^{\prime}(\operatorname{conv}(S))=\{f \in$ $\left.\mathcal{F}_{j}(\operatorname{conv}(S)) \mid 0 \in f\right\}$. Let a face $f \in \mathcal{F}_{j}^{\prime}(\operatorname{conv}(S))$ be defined by $f=H \cap \operatorname{conv}(S)$ with a supporting half-plane $H$. As $H$ also supports $C(S)$ at the origin, a subset $g=H \cap C(S)$ of $C(S)$ is a $j$-dimensional face of $C(S)$. It is easy to show that this correspondence between a face in $\mathcal{F}_{j}^{\prime}(\operatorname{conv}(S))$ and one in $\mathcal{F}_{j}(C(S))$ is oneto-one and preserves the relation $\prec$. It implies that the structure of abstract complex of $\mathcal{F}(C(S))$ is the same as one of $\mathcal{F}^{\prime}(\operatorname{conv}(S))$. As the result, we obtain the following algorithm of constructing $C(S)$ in $\mathbb{R}^{m}$ :

1. Construct $\mathcal{F}(\operatorname{conv}(S))$ from $S$ by a convex hull algorithm for a polytope.

2. If $0 \notin \mathcal{F}_{0}(\operatorname{conv}(S))$ then we ignore $C(S)^{\vee}$, since it does not contribute to the integration.

3. Construct a subset of faces $\mathcal{F}^{\prime}(\operatorname{conv}(S))$ selecting ones including the origin.

4. Interpret $\mathcal{F}^{\prime}(\operatorname{conv}(S))$ as the set of faces $\mathcal{F}(C(S))$.

The dual cone of $C(S)$ is constructed from $\mathcal{F}(C(S))$ by the dual operation shown by Proposition A.6 of Ref. [15. In our case, it is sufficient to know the set of edges $\mathcal{F}_{1}\left(C(S)^{\vee}\right)$, since $C(S)^{\vee}$ is expressed by:

$$
C(S)^{\vee}=\left\{\sum_{u \in \mathcal{F}_{1}\left(C(S)^{\vee}\right)} \mu_{u} u \mid \mu_{u} \in \mathbb{R}_{\geq 0}, \forall u \in \mathcal{F}_{1}\left(C(S)^{\vee}\right)\right\}
$$

where $u \in \mathcal{F}_{1}\left(C(S)^{\vee}\right)$ means to select a non-zero vector $u$ for each edge such that $u$ lies on the edge. If $\operatorname{dim} C(S)=m$, an edge of $C(S)^{\vee}$ is obtained as a half-line starting from the origin and parallel to the normal vector of a facet of $C(S)$. If $\operatorname{dim} C(S)<m, C(S)^{\vee}$ is not strongly convex and the construction of $\mathcal{F}_{1}\left(C(S)^{\vee}\right)$ is slightly complicated. However, we do not need to consider this case, as discussed before.

We show that when $S$ is a set of integer vectors, a vector $u \in \mathcal{F}_{1}\left(C(S)^{\vee}\right)$ can be selected as an integer vector. Since vertices of $\operatorname{conv}(S)$ are included in the original set of points $S$, we can select integer vectors to represent the edges of $C(S)$. A facet of $C(S)$ is a subset of hyperplane spanned by some of these vectors. A vector representing an edge of $C(S)^{\vee}$ is a vector normal to the facet. The normal vector is obtained by solving a system of homogeneous linear equations. The vector $u$ is solved as a vector $w$ multiplied by the inverse matrix 
of an integer matrix $M$. The inverse matrix is constructed by the minor of $M$ divided by the determinant $\operatorname{det} M$, where the elements of the minor are integers. By adjusting the normalization of vector $w$, we can cancel the denominator $\operatorname{det} M$. In this way, we can take $v$ as an integer vector.

As the result, $\Delta_{b b^{\prime}}$ can be expressed with a set of integer vectors $\left\{v_{1}, \cdots, v_{m}\right\} \subset$ $\mathbb{Z}_{\geq 0}^{N-1}$ by:

$$
\Delta_{b b^{\prime}}=\left\{\sum_{i} r_{i} v_{i} \mid r_{i} \geq 0\right\}
$$

With the fact that our set of points $Z_{b b^{\prime}}$ includes $E^{N-1}$ as a subset, we can accelerate the algorithm in the following cases:

- Case where there exists a non-zero vector $v \in Z_{b}^{\mathcal{U}_{l}} \cup Z_{b^{\prime}}^{\mathcal{F}_{l}}$ and $v \in \mathbb{R}_{\leq 0}^{N-1}$. In this case, negated vector $-v$ is expressed by a linear combination of vectors in $E^{N-1}$ with non-negative coefficients. It means that $C\left(Z_{b}^{\mathcal{U}_{l}} \cup Z_{b^{\prime}}^{\mathcal{F}_{l}} \cup E^{N-1}\right)$ includes line segment connecting $v$ and $-v$ and thus the origin is not the vertex. So we can discard such a configuration.

- Case where there exists a non-zero vector $v \in Z_{b}^{\mathcal{U}_{l}} \cup Z_{b^{\prime}}^{\mathcal{F}_{l}}$ and $v \in \mathbb{R}_{>0}^{N-1}$. In this case, vector $v$ is an interior point of $C\left(Z_{b}^{\mathcal{U}_{l}} \cup Z_{b^{\prime}}^{\mathcal{F}_{l}} \cup E^{N-1}\right)$ and is not on the edge of the cone. So we can eliminate $v$ from the input set of points. That is, the input set of points $Z_{b}^{\mathcal{U}_{l}} \cup Z_{b^{\prime}}^{\mathcal{F}_{l}} \cup E^{N-1}$ can be replaced by $\left\{\left(Z_{b}^{\mathcal{U}_{l}} \cup Z_{b^{\prime}}^{\mathcal{F}_{l}}\right) \backslash \mathbb{R}_{\geq 0}^{N-1}\right\} \cup\{0\} \cup E^{N-1}$. This replacement decreases the number of input points, which accelerate the convex hull algorithm.

These conditions are easily checked before applying the convex hull algorithm.

\subsection{Triangulation of convex polyhedral cones}

We discuss here how to triangulate convex polyhedral cones. Since a convex polyhedral cone is not bounded, we convert it to a bounded polytope in order to make the problem easier to handle. Let $H$ be a hyperplane perpendicular to vector $\mathbb{I}:=(1,1, \cdots, 1)$ and passing through a point $w \mathbb{I}$ for $w \in \mathbb{R}_{>0}$. As $\Delta_{b b^{\prime}} \subset \mathbb{R}_{>0}^{N-1}$, this cone can be truncated to a polytope in cutting by $H$. Then the resulting polytope $\Delta_{b b^{\prime}}^{\prime}$ is expressed by:

$$
\begin{aligned}
\Delta_{b b^{\prime}}^{\prime} & =\left\{\sum_{j} r_{j} v_{j}^{\prime} \mid 0 \leq r_{j} \leq 1, \sum_{j} r_{j} \leq 1\right\}, \\
v_{j}^{\prime} & :=v_{j} w /\left(\mathbb{I}, v_{j}\right), \quad\left(\mathbb{I}, v_{j}^{\prime}\right)=w .
\end{aligned}
$$

Since $v_{j}$ is an integer vector, $\left(\mathbb{I}, v_{j}\right)$ is integer. By selecting $w$ as the least common multiplier $\operatorname{lcm}\left\{\left(\mathbb{I}, v_{j}\right) \mid \forall j\right\}$, we can take all of $\left\{v_{j}^{\prime}\right\}$ as integer vectors.

The triangulation of $(N-1)$-dimensional polytope is not evident. We restrict ourselves to triangulation methods such that no new vertices are added. Even with this restriction, the number of resulting simplicies is not unique. The following example (Fig. 2) shows the case where the different numbers of simplicies are produced by different triangulations. Let $A, B, C, D, E$ be points in the 3-dimensional space defined by:

$$
\begin{aligned}
& A=(0,0,0), \quad B=(1,0,1), \quad C=(1,1,1), \\
& D=(0,1,1), \quad E=(0,0,2) .
\end{aligned}
$$




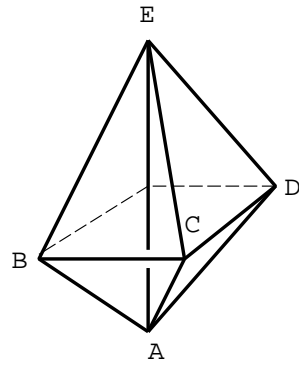

(a)

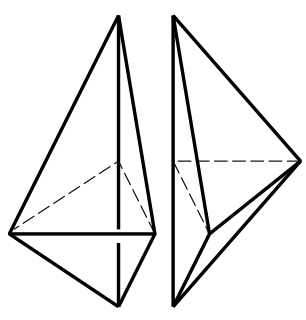

(b)

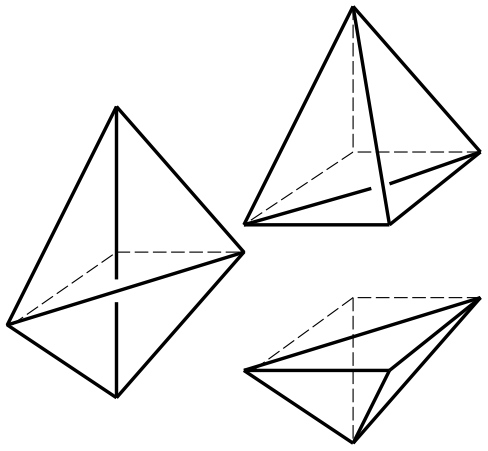

(c)

Figure 2: An example of different triangulations

The convex hull of these points is shown by Fig. 2(a). In cutting the convex hull by a triangle $(A, C, E)$, we obtain two simplicies (Fig. 2(b)). On the other hand, there are three simplicies when it is cut by three triangles $(A, B, D),(E, B, D)$ and $(B, C, D)$ (Fig. 2(c)). We do not know triangulation algorithms which produce the minimum number of simplicies. We use the following algorithm.

1. If the number of vertices of the input $d$-dimensional polytope $P$ is $d+1$ then $P$ is a simplex. The algorithm returns the set $\{P\}$. This condition is always satisfied when $d=1$ ( $P$ is a line segment).

2. Pick up one vertex $u$ from $P$. Remove $u$ and all interior points of all line segments included in $P$ and starting from $u$. The resulting geometric object is the union of polytopes $Q_{j}(j=1, \cdots, m)$ of dimension $d-1$, which are $(d-1)$-dimensional faces not including $u$.

3. For all $j=1, \cdots, m$, apply triangulation algorithm to $Q_{j}$ recursively, and set $S_{j}$ of simplicies is obtained.

4. Make the union $S=\cup_{j} S_{j}$.

5. Construct a $d$-dimensional simplicies by adding $u$ to each $(d-1)$-dimensional simplex in $S$. The algorithm returns the set of all constructed simplicies.

It is noted that this algorithm never falls into the infinite loop. Since step 2 removes at least one face, the number of recusive applications of the algorithm is bounded by the number of faces of the polytope. The number of resulting simplicies depends on how vertex $u$ is selected in step 2. One choice is to select $u$ when it attaches the maximum number of edges. This condition produces relatively smaller number of resulting simplicies, which result will be shown in the next section.

For the triangulation of a cone, one must select the origin for $u$ in the step 2 at the first time before calling subsequent recursive procedure. This selection guarantees that each simplex includes the origin as one of its vertices. Let $s_{v}$ be one of the obtained simplicies and characterized by a set of $N-1$ linearly 
independent vectors $\left\{v_{j}\right\}$ as:

$$
s_{v}=\left\{\sum_{j} r_{j} v_{j} \mid \sum_{j} r_{j} \leq 1,0 \leq r_{j}(\forall j)\right\} .
$$

From this simplex, a simplicial cone $s_{v}^{\prime}$ is obtained by extending the range of the values of $\left\{r_{j}\right\}$ :

$$
s_{v}^{\prime}=\left\{\sum_{j} r_{j} v_{j} \mid 0 \leq r_{j}(\forall j)\right\}
$$

It is noted that vector $v_{j}$ is an integer vector, for it is one of the vertices in the polytope before triangulation.

\section{A test implementation}

We have prepared a program in order to confirm the correctness of our method. For the convex hull method we adopted incremental algorithm described in Ref. [16] and modified it for convex polyhedral cones. In order to avoid the accuracy problem of numerical data, all numerical operation are performed in integer arithmetic. We adopted program language python which is equipped with arbitrary long integer arithmetic. Input data is prepared by the program package described in Ref. [10. The output integrand of our program is passed to the same program package, which continues the subsequent divergence separation and integration steps.

The procedure of constructing convex hull is compared with qhull package [17. The triangulation procedure is checked by computing the volume of resulting simplicies. Integrated values are compared with ones in published articles in several cases. We show the number of decomposed sectors in Table 1 Column " $\mathrm{H}$ " is cited from Ref. [6] and columns "A", "B", "C", "S" and "X" from Ref. [13]

Table 1: The number of sectors

\begin{tabular}{|l|ccccc|c|c|}
\hline Diagram & A & B & C & S & X & H & $\begin{array}{c}\text { This } \\
\text { method }\end{array}$ \\
\hline Bubble & 2 & 2 & 2 & - & 2 & - & 2 \\
Triangle & 3 & 3 & 3 & - & 3 & - & 3 \\
Box & 12 & 12 & 12 & 12 & 12 & - & 12 \\
\hline Tbubble & 58 & 48 & 48 & - & 48 & - & 48 \\
Double box, $p_{i}^{2}=0$ & 775 & 586 & 586 & 362 & 293 & 282 & 266 \\
Double box, $p_{4}^{2} \neq 0$ & - & - & - & - & - & 197 & 186 \\
Double box, $p_{i}^{2}=0$ & 1138 & 698 & 698 & - & 395 & - & 360 \\
$\quad$ nonplanar & & & & & & & \\
D420 & 8898 & 564 & 564 & 180 & $\mathrm{~F}$ & - & 168 \\
\hline 3 loop vertex (A8) & - & - & - & - & - & 684 & 684 \\
Triple box & $\mathrm{M}$ & 114256 & 114256 & 22657 & 10155 & - & 6568 \\
\hline
\end{tabular}

This test program is too slow for the practical purposes. We are planning to prepare a practical implementation in $\mathrm{C}++$. 


\section{Conclusion}

We proposed a new method of IR factorization in sector decomposition employing a geometric interpretation of the problem. The original problem is converted into a set of problems in convex geometry: (1) construction of intersection among dual cones of convex polyhedral cones corresponding to each polynomial in the original problem and (2) triangulation of them. They are solved by the algorithms in combinatorial geometry. This is a deterministic method and never falls into a infinite loop. The number of resulting sectors depends on the algorithm of triangulation. Our test implementation of this method shows the smaller number of sectors compared to other methods based on iterated decomposition.

\section{Acknowledgments}

The authors wish to express their thanks to the members of Minami-tateya group for the useful discussions. This work is supported in part by Ministry of Education, Science, and Culture, Japan under Grant-in-Aid No.20340063 and No.21540286.

\section{References}

[1] Klaus Hepp. "Proof of the bogoliubov-parasiuk theorem on renormalization," Commun. Math. Phys. 2 (1966) 301-326.

[2] M. Roth and A. Denner, "High-energy approximation of one-loop Feynman integrals," Nucl. Phys. B 479 (1996) 495 [arXiv:hep-ph/9605420].

[3] T. Binoth and G. Heinrich, "An automatized algorithm to compute infrared divergent multi-loop integrals," Nucl. Phys. B 585 (2000) 741 arXiv:hep-ph/0004013.

[4] T. Binoth and G. Heinrich, "Numerical evaluation of multi-loop integrals by sector decomposition," Nucl. Phys. B 680 (2004) 375 arXiv:hep-ph/0305234.

[5] T. Binoth and G. Heinrich, "Numerical evaluation of phase space integrals by sector decomposition," Nucl. Phys. B 693 (2004) 134 arXiv:hep-ph/0402265.

[6] G. Heinrich, "Sector Decomposition," Int. J. Mod. Phys. A 23 (2008) 1457 arXiv:0803.4177 [hep-ph]].

[7] A. Lazopoulos, K. Melnikov and F. Petriello, "QCD corrections to tri-boson production," Phys. Rev. D 76 (2007) 014001 arXiv:hep-ph/0703273.

[8] C. Anastasiou, S. Beerli and A. Daleo, "Evaluating multi-loop Feynman diagrams with infrared and threshold singularities numerically," JHEP 0705 (2007) 071 arXiv:hep-ph/0703282.

[9] V. Pilipp, "Semi-numerical power expansion of Feynman integrals," JHEP 0809 (2008) 135 arXiv:0808.2555 [hep-ph]]. 
[10] T. Ueda and J. Fujimoto, "New implementation of the sector decomposition in FORM," PoS (ACAT08) 120, 2008 arXiv:0902.2656 [hep-ph].

[11] C. Bogner and S. Weinzierl, "Resolution of singularities for multi-loop integrals," Comput. Phys. Commun. 178 (2008) 596 arXiv:0709.4092 [hep$\mathrm{ph}]$.

[12] C. Bogner and S. Weinzierl, "Blowing up Feynman integrals," Nucl. Phys. Proc. Suppl. 183 (2008) 256 arXiv:0806.4307 [hep-ph]].

[13] A. V. Smirnov and M. N. Tentyukov, Comput. Phys. Commun. 180 (2009) 735 arXiv:0807.4129 [hep-ph]].

[14] A. V. Smirnov and V. A. Smirnov, "Hepp and Speer Sectors within Modern Strategies of Sector Decomposition," JHEP 0905 (2009) 004 arXiv:0812.4700 [hep-ph].

[15] Tadao Oda. "Convex Bodies and Algebraic Geometry." Springer-Verlag, 1987.

[16] H. Edelsbrunner. Algorithms in Combinatorial Geometry. Springer, 1987.

[17] Program package qhull, http://www.qhull.org/.

[18] For the list of program packages, see, for example, http://www.geom. uiuc.edu/software/cglist/. 